\title{
Maternal butyrate supplementation induces insulin resistance associated with enhanced intramuscular fat deposition in the offspring
}

\author{
Yanping Huang ${ }^{1}$, Shixing Gao ${ }^{1}$, Jinglong Chen ${ }^{1}$, Elke Albrecht ${ }^{2}$, Ruqian Zhao ${ }^{1}$, \\ Xiaojing Yang ${ }^{1}$ \\ ${ }^{1}$ Key Laboratory of Animal Physiology \& Biochemistry, Nanjing Agricultural University, Nanjing 210095, P. R. China \\ ${ }^{2}$ Leibniz Institute for Farm Animal Biology, Institute for Muscle Biology and Growth, Dummerstorf, Germany \\ Correspondence to: Xiaojing Yang, email: yangxj@njau.edu.cn \\ Keywords: maternal butyrate, insulin resistance, epigenetic regulation, offspring skeletal muscle \\ Received: September 20, 2016 \\ Accepted: December 01, 2016 \\ Published: December 30, 2016
}

\section{ABSTRACT}

Maternal nutrition is important for the risk of the offspring to develop insulin resistance and adiposity later in life. The study was undertaken to determine effects of maternal butyrate supplementation on lipid metabolism and insulin sensitivity in the offspring skeletal muscle. The offspring of rats, fed a control diet or a butyrate diet ( $1 \%$ sodium butyrate) throughout gestation and lactation, was studied at weaning and at $\mathbf{6 0}$ days of age. The offspring of dams fed a butyrate diet had higher HOMAinsulin resistance and impaired glucose tolerance. This was associated with elevated mRNA and protein expressions of lipogenic genes and decreased amounts of lipolytic enzyme. Simultaneously, enhanced acetylation of histone H3 lysine 9 and histone H3 Iysine 27 modification on the lipogenic genes in skeletal muscle of adult offspring was observed. Higher concentration of serum insulin and intramuscular triglyceride in skeletal muscle of offspring from the butyrate group at weaning were accompanied by increasing levels of lipogenic genes and enrichment of acetylation of histone H3 Iysine 27. Maternal butyrate supplementation leads to insulin resistance and ectopic lipid accumulation in skeletal muscle of offspring, indicating the importance of short chain fatty acids in the maternal diet on lipid metabolism.

\section{INTRODUCTION}

Maternal nutrition during gestation and lactation has profound effects on fetal growth and development with lifelong consequences [1,2]. Animal models confirm that maternal diet influences the development of insulin resistance and adiposity in the offspring [3,4]. Insulin resistance is a well-recognized factor in the development and progression of a series of disorders including type II diabetes and hypertension, dyslipidemia, and cardiovascular disease [5]. It has also been shown that nutritional factors during offspring early life possibly influence permanently the risk of an individual to develop insulin resistance in adulthood $[6,7]$.

Maternal dietary fatty acids not only are important as an energy source but also play important role in regulation of gene expression in cells and in intercellular communication in offspring $[1,8]$. Currently, most studies focus on the effects of long-chain fatty acids rather than on short chain fatty acids (SCFA) [8]. Butyrate is a SCFA which is produced in the gastrointestinal tract [9], and acts as an energy source to meet energy requirements in ruminants and monogastric animals [10, 11]. Besides, butyrate acts as a histone deacetylase (HDAC) inhibitor which can inhibit deacetylation of histone and various transcription factors, involved in several biological pathways associated with the pathogenesis of diabetes [12, 13]. In vivo, several studies have shown that butyrate can alleviate high fat diet-induced obesity and improve insulin sensitivity in skeletal muscle of mice under a high fat diet $[14,15]$. However, a previous study suggested maternal high fiber diet, which could produce butyrate by microbial fermentation in the gastrointestinal tract, increased the expressions of CCAAT/enhancer-binding protein (C/EBP) and peroxisome proliferator-activated receptor $\gamma(\operatorname{PPAR} \gamma)$ related to adipogenesis [16]. Additionally, Radunz et al. [17] found that progeny from dams fed high fiber diet had increased intramuscular fat deposition and diminished 
Table 1: Growth performance of weaning and adult offspring of rats fed either a control or butyrate supplemented diet throughout gestation and lactation

\begin{tabular}{lccc}
\hline Variables & Control $(\mathbf{n}=\mathbf{8})$ & Butyrate $(\mathbf{n}=\mathbf{8})$ & P-value \\
\hline Weaning rats & & & \\
Body weight $(\mathrm{BW})(\mathrm{g})$ & $69.23 \pm 2.04$ & $73.17 \pm 1.02$ & 0.10 \\
Gastrocnemius muscle weight $(\mathrm{GW})(\mathrm{g})$ & $0.46 \pm 0.03$ & $0.48 \pm 0.02$ & 0.43 \\
$\mathrm{GW}: \mathrm{BW}(\mathrm{g} / \mathrm{kg})$ & $6.60 \pm 0.24$ & $6.60 \pm 0.26$ & 0.99 \\
Adult rats & & & \\
Body weight $(\mathrm{BW})(\mathrm{g})$ & $247.89 \pm 4.51$ & $3.37 \pm 0.12$ & $<0.01$ \\
Gastrocnemius & $2.92 \pm 0.06$ & $11.66 \pm 0.27$ & $<0.01$ \\
muscle weight $(\mathrm{GW})(\mathrm{g})$ & $11.18 \pm 0.64$ & 0.52 \\
GW : BW $(\mathrm{g} / \mathrm{kg})$ &
\end{tabular}

The values are presented as mean \pm SEM.

insulin response. In vitro, butyrate was found to stimulate adipogenesis and triglyceride storage $[18,19]$. Whether maternal butyrate supplementation could have effects on lipid metabolism and insulin sensitivity in the offspring skeletal muscle has never been shown.

The ectopic accumulation of lipids in skeletal muscle has been viewed as a major factor in the etiology of insulin resistance and type 2 diabetes by reducing insulin stimulated glucose uptake [20-23]. During the past two decades, increasing emphasis was placed on understanding the connection between intramuscular triacylglycerol content and insulin action. Interest in this topic grew from numerous reports showing a strong negative association between intramuscular triacylglycerol content and insulin sensitivity [24, 25]. This relationship was also evident in obese and diabetic humans as well as in several rodent and cell culture models of metabolic disease and/or chronic lipid exposure [26-28]. However, the mechanism, how maternal butyrate supplementation influences the relationship between lipid metabolism and insulin sensitivity, is largely unclear.

In the present study, we used the maternal sodium butyrate supplement during pregnancy and lactation in rat as a model to investigate the offspring skeletal muscle lipid deposition and possible consequences for the insulin sensitivity. The results will further contribute to clarify the effect of maternal short chain fatty acids on offspring metabolism.

\section{RESULTS}

\section{Maternal butyrate increased offspring body and muscle weight}

The body weight and gastrocnemius muscle weight (GW) $(\mathrm{P}<0.01)$ of adult offspring were significantly enhanced in the butyrate group compared with control (Table 1), though both of them showed no obvious differences at weaning age.

\section{Impaired insulin sensitivity in offspring of butyrate supplemented rats}

Serum concentrations of insulin and NEFA were increased $(\mathrm{P}<0.05)$ at weaning in offspring of butyrate supplemented rats (Table 2). This difference was not observed in adult offspring. However, concentration of fasting glucose was higher $(\mathrm{P}<0.05)$ in the butyrate group (Table 2). When adult offspring were challenged with a GTT and an ITT, butyrate group rats showed permanently increased serum glucose levels (Figure $1 \mathrm{a}, 1 \mathrm{~b}$ ), indicating decreased responsiveness as a sign for development of insulin resistance. Furthermore, HOMA-IR (HOMA-insulin resistance) was significantly increased in both weaning offspring $(\mathrm{P}<0.01)$ and adult offspring ( $\mathrm{P}<0.05)$ belonging to the butyrate group (Table 2).

\section{Maternal butyrate inhibited protein expressions related to the insulin signaling and glucose uptake $n$ skeletal muscle from adult offspring}

Because of the whole body insulin resistance observed in the adult offspring, we examined components of the insulin-signaling pathway in these animals. Phosphorylation of IRS at ser1101 was increased $(\mathrm{P}<$ $0.05)$ and the abundances of total PI3K/P85 $(\mathrm{P}<0.05)$ and total AKT protein $(\mathrm{P}=0.07)$ were reduced in skeletal muscle of the butyrate group (Figure 2). With the higher concentration of fasting glucose in adult rats, GLUT 4 related to glucose uptake in skeletal muscle was decreased $(\mathrm{P}<0.05)$ in the butyrate group (Figure 2). 
Table 2: Serum glucose, total triglyceride content and intramuscular triglyceride content in skeletal muscle of weaning and adult offspring

\begin{tabular}{lccccccc}
\hline & \multicolumn{3}{c}{ Weaning rats } & & \multicolumn{3}{c}{ Adult rats } \\
\cline { 2 - 3 } & $\begin{array}{c}\text { Control } \\
(\mathbf{n}=\mathbf{8})\end{array}$ & $\begin{array}{c}\text { Butyrate } \\
(\mathbf{n}=\mathbf{8})\end{array}$ & P-value & & $\begin{array}{c}\text { Control } \\
(\mathbf{n}=\mathbf{8})\end{array}$ & $\begin{array}{c}\text { Butyrate } \\
(\mathbf{n}=\mathbf{8})\end{array}$ & P-value \\
\hline Serum Glucose $(\mathrm{mmol} / \mathrm{L})$ & $7.21 \pm 0.26$ & $6.84 \pm 0.16$ & 0.80 & $10.36 \pm 0.77$ & $13.03 \pm 0.76$ & $<0.05$ \\
Serum Insulin $(\mu \mathrm{U} / \mathrm{ml})$ & $10.87 \pm 2.30$ & $16.41 \pm 1.96$ & $<0.05$ & $13.32 \pm 1.46$ & $16.61 \pm 2.35$ & 0.20 \\
Serum Cholesterol $(\mathrm{mmol} / \mathrm{L})$ & $2.98 \pm 0.18$ & $2.93 \pm 0.09$ & 0.22 & $1.51 \pm 0.06$ & $1.77 \pm 0.27$ & 0.44 \\
Serum Triglyceride $(\mathrm{mmol} / \mathrm{L})$ & $2.22 \pm 0.48$ & $1.82 \pm 0.21$ & 0.79 & $2.08 \pm 0.44$ & $1.84 \pm 0.17$ & 0.89 \\
Serum NEFA $(\mu \mathrm{mol} / \mathrm{L})$ & $441.6 \pm 36.3$ & $634.2 \pm 55.0$ & $<0.05$ & & $590.3 \pm 28.5$ & $569.3 \pm 56.4$ & 0.73 \\
IntramuscularTriglyceride & $1.99 \pm 0.18$ & $3.50 \pm 0.49$ & $<0.05$ & & $1.46 \pm 0.67$ & $4.38 \pm 1.08$ & $<0.05$ \\
(mg/g) & $2.29 \pm 0.23$ & $4.87 \pm 0.46$ & $<0.01$ & & $3.31 \pm 0.42$ & $4.89 \pm 0.66$ & $<0.05$ \\
\hline HOMA-IR & & & & & &
\end{tabular}

The values are presented as mean \pm SEM.

a
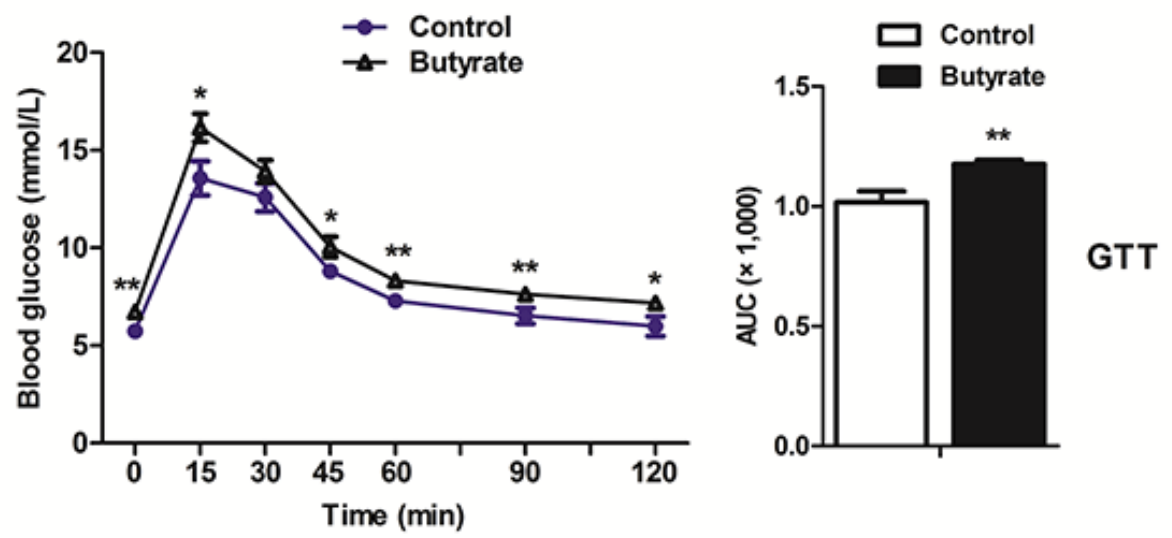

b
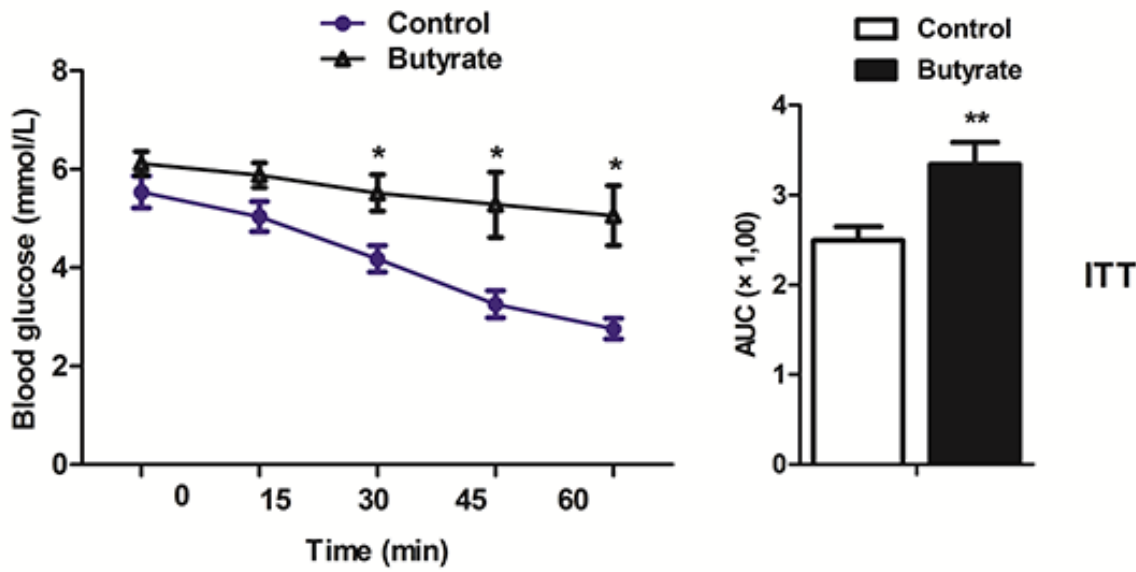

Figure 1: Maternal butyrate supplementation throughout gestation and lactation induced the whole body insulin resistance as indicated in glucose tolerance test $a .(n=10$ in controls; $n=12$ in butyrate group) and insulin tolerance test $\mathbf{b}$. $(\mathbf{n}=\mathbf{5}$ per group) in adult offspring. $* \mathrm{P}<0.05$, $* * \mathrm{P}<0.01$, compared with control. 


\section{Maternal butyrate increased intramuscular triglyceride content of offspring}

Serum total cholesterol and total triglyceride were not affected in the offspring at weaning by butyrate supplementation of the dams $(\mathrm{P}>0.05)$. However, significantly higher $(\mathrm{P}<0.05)$ content of intramuscular triglyceride was observed in the butyrate group at weaning (Table 2).

Later in life, total cholesterol and triglyceride in serum were also not different between the butyrate group and the control group, while the content of intramuscular triglyceride further increased significantly $(\mathrm{P}<0.05)$ in the butyrate group (Table 2). Oil-red O staining revealed the abundance of intramuscular adipocytes in muscle cross sections of butyrate group rats but not in that of controls (Supplementary Figure 1).

\section{mRNA and protein abundance of lipid metabolism related genes in gastrocnemius muscle}

Among these lipid related genes, PPAR $\gamma$ mRNA expression was higher $(\mathrm{P}<0.05)$ in skeletal muscle of weaning rats in the butyrate group, which was consistent with a significant up-regulation of PPAR $\gamma$ protein expression $(\mathrm{P}<0.05)$. Although $\mathrm{C} / \mathrm{EBP} \beta$ and FAS mRNA expressions in weaning rats were not altered, their protein levels were higher $(\mathrm{P}<0.05)$ in the butyrate group. On the other hand, PNPLA2 (ATGL) mRNA expression related to lipolysis was significantly decreased $(\mathrm{P}<0.05)$ (Figure $3 a)$ while its protein level was still higher $(\mathrm{P}<0.05)$ in weaning rats of the butyrate group (Figure $3 b$ ).

During the offspring adult life, lipid related transcription factors including PPAR $\gamma$ and $\mathrm{C} / \mathrm{EBP} \beta$ mRNA (Figure $4 \mathrm{a}$ ) and protein abundances (Figure $4 \mathrm{~b}$ ) were enhanced $(\mathrm{P}<0.05)$ in gastrocnemius muscle of female rats in the butyrate group. Among the genes involved in lipolysis, PNPLA2 and HSL mRNA expression did not change (Figure 4a) whereas the ATGL protein level (P $<0.05)$ and the phosphorylation level of HSL $(\mathrm{P}<0.05)$ decreased (Figure $4 b$ ). FAS mRNA (Figure $4 a$ ) and protein expression (Figure $4 \mathrm{~b}$ ) in adult rats showed no obvious changes between two groups.

\section{Histone modifications on the promoter of lipid metabolism related genes}

In the gastrocnemius muscle of weaning offspring, higher enrichment $(\mathrm{P}<0.05)$ of acH3K27 was found for PPAR $\gamma$ and a trend for higher enrichment $(\mathrm{P}=0.07)$ for $\mathrm{C} /$ EBP $\beta$ in the butyrate group. Histone modification on the promoter of the FAS gene was not influenced by maternal butyrate supplementation (Figure 5a).

Furthermore, in the gastrocnemius muscle of adult offspring, maternal butyrate supplementation during gestation and lactation significantly enhanced the enrichment of acH3K9 and acH3K27 on the promoters of $\mathrm{C} / \mathrm{EBP} \beta(\mathrm{P}<0.05)$ and FAS $(\mathrm{P}<0.05)$ (Figure 5b, 5c). Furthermore, we observed enrichment of acH3K27 on the promoter of PPAR $\gamma$ (Figure 5c).

\section{DISCUSSION}

The current study highlights the importance of maternal nutrition for the offspring phenotype. It is the first to indicate that maternal butyrate supplementation during gestation and lactation induces insulin resistance in adult female offspring. The results show that insulin resistance could be induced by the accumulation of TG in skeletal muscle. Furthermore, the mechanisms involved in mediating the effects of maternal butyrate on the
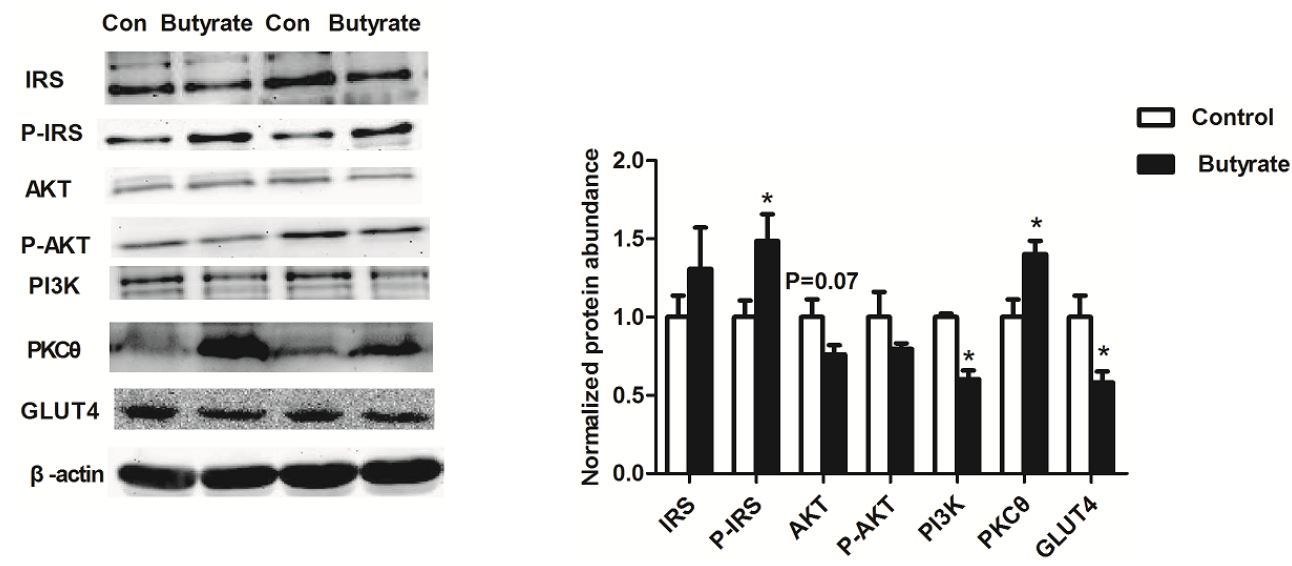

Figure 2: Effect of maternal butyrate supplementation throughout gestation and lactation on protein expression of insulin signaling molecules and GLUT4 protein expression in skeletal muscle of adult offspring $(\mathrm{n}=6$ per group). $\beta$-actin was used for normalization. Values are mean \pm SEM. $* \mathrm{P}<0.05$, compared with control. 
intramuscular fat deposition include increasing lipogenic and reducing lipolysis genes in offspring skeletal muscle partly via enhanced histone acetylation modification.

Maternal nutrition can influence the physiology and metabolism of the offspring such as insulin sensitivity $[30,31]$. Prior studies have shown that offspring from dams fed high fiber diet, which could produce butyrate by microbial fermentation in the gastrointestinal tract, had diminished insulin response [17]. In the present study, the adult offspring that were born to dams on butyrate diet had higher serum glucose, HOMA-IR and impaired glucose tolerance, suggesting that they were developing insulin resistance.

Skeletal muscle is a primary site of glucose disposal and insulin action [32]. Many insulin responses are activated through the PI3-kinase/Akt pathway [33, 34]. The increased Ser1101-IRS1 phosphorylation and decreased PI3K p $85 \alpha$ subunit and AKT showed that the insulin signaling was impaired in skeletal muscle from adult offspring in butyrate group. Increased Ser1101 phosphorylation of IRS1 has been shown to inhibit IRS1 function and could be a specific target of PKC $\theta$ [35]. The PKC $\theta$ promotes IRS-1 serine phosphorylation and impaired downstream insulin signaling [20, 35]. A higher protein expression of $\mathrm{PKC} \theta$ was observed in skeletal muscle of adult offspring of the butyrate group in our research. Glucose uptake could be prevented by inhibiting the PI3K/Akt pathway [34]. Previous studies showed that maternal obesity during the periconceptional period resulted in lower GLUT4 protein abundance in skeletal muscle in female lambs [36]. We found maternal butyrate supplementation inhibited the protein expression of PI3K/ p85 and GLUT4 in skeletal muscle in adult offspring. Our data indicate for the first that maternal butyrate diet can induce whole-body insulin resistance and inhibits insulin signaling pathway and glucose uptake in skeletal muscle in adult offspring.

Several studies have shown that a high fat diet with butyrate supplementation could alleviate obesity and improve insulin sensitivity in skeletal muscle of mice $[14,15]$. However, in the present study, we observed that maternal normal diet with butyrate supplementation has increased the levels of intramuscular triglycerides in both weaning and adult offspring skeletal muscle. In addition, our previous study demonstrated maternal butyrate supplementation led to lipid accumulation in offspring

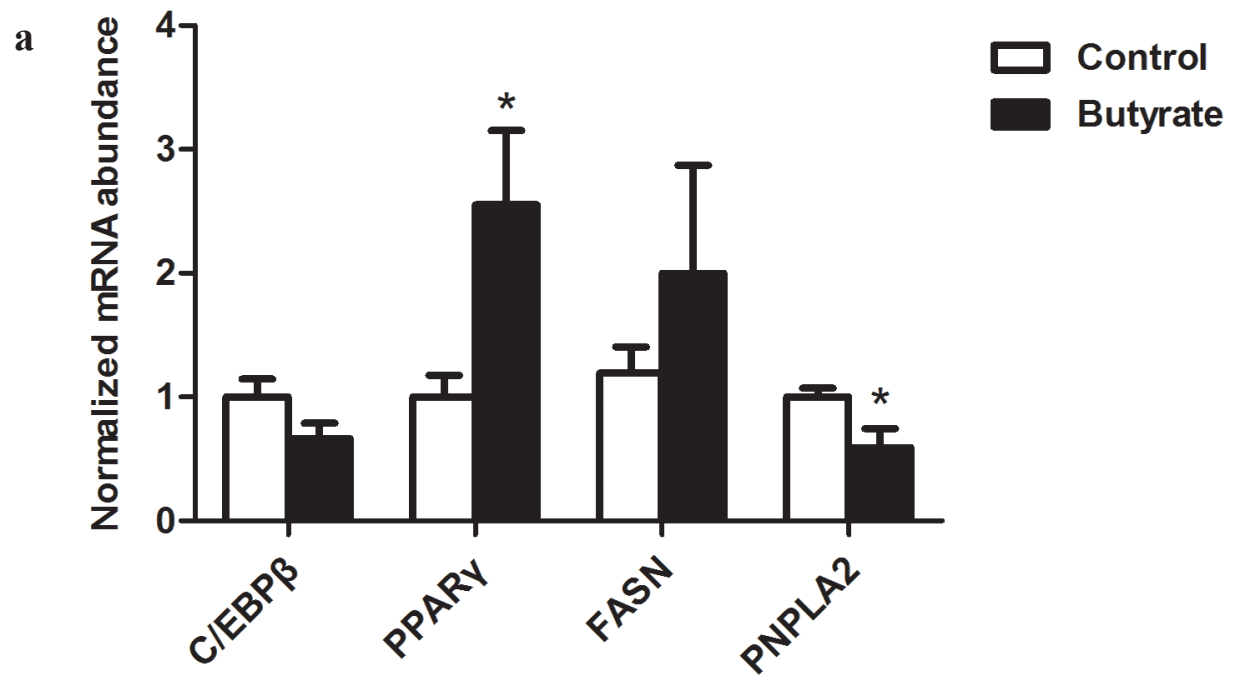

b

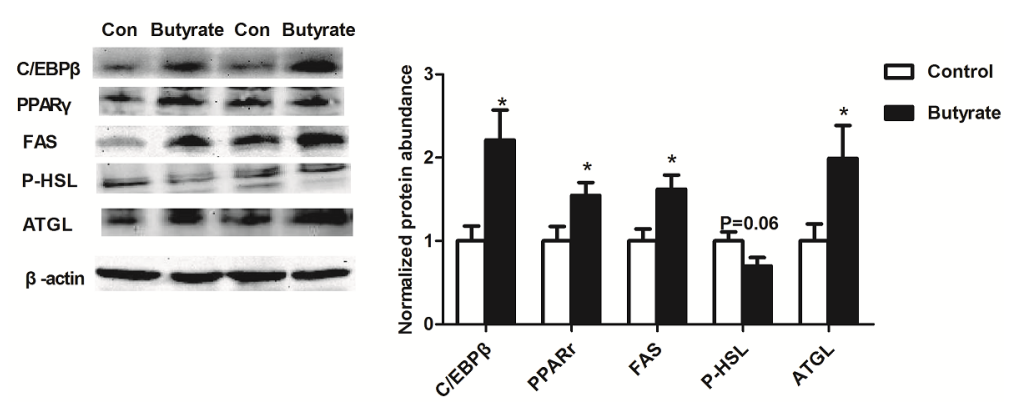

Figure 3: Effect of maternal butyrate supplementation throughout gestation and lactation on $\mathrm{mRNA}$ a. $(\mathrm{n}=8$ per group) and protein expression $b .(n=6$ per group) of genes related to lipid metabolism in skeletal muscle of weaning offspring. $\beta$-actin was used for normalization. Values are mean $\pm \mathrm{SEM}$. ${ }^{*} \mathrm{P}<0.05$, compared with control. 
liver of weaning-age rats [37]. These differences suggest that maternal normal diets with butyrate supplementation induced the onset of intramuscular triglyceride deposition in offspring at early age, indicating the importance of maternal nutrition environment.

Furthermore, in porcine stromal-vascular cells, butyrate was found to stimulate adipocyte differentiation and stimulation of triglyceride storage $[18,19]$. In addition to lipid accumulation within myocytes, adipocytes developed in close proximity to myocytes, induced by upregulation of key regulating transcription factors like PPAR $\gamma$ and $\mathrm{C} / \mathrm{EBP} \beta$ [38-42]. Thus, to illustrate intramuscular triglyceride deposition, in our present study the increased expressions of C/EBP $\beta$ and PPAR $\gamma$ were found. Similarly, butyrate was reported to increase lipid accumulation including increasing the levels of transcriptional factors like C/EBPs and PPAR $\gamma$ in preadipocytes $[18,43]$.

Given the fact that ATGL and HSL are responsible for more than $95 \%$ of the TAG hydrolase activity [44], reduced ATGL and HSL may contribute to accumulation of intramuscular triglycerides and insulin resistance. In the present study, at adulthood, HSL phosphorylation and ATGL were dramatically reduced in the skeletal muscle of the butyrate group. At weaning age, ATGL protein expression was up-regulated and HSL phosphorylation tended to decrease with maternal butyrate supplementation. Previous studies have also shown that up-regulation of ATGL expression and/or inhibition of HSL activity promoted diacylglycerols accumulation in skeletal muscle and impaired insulin signaling [23, 45]. Taken together, the data suggests that maternal butyrate supplementation induced accumulation of intramuscular triglycerides in offspring skeletal muscle may be caused at least in part by a reduced expression of HSL and ATGL.

As shown before, butyrate has HDAC inhibitory activity [46-48]. The activation of histone acetylation could increase the transcriptional regulation of lipogenetic and metabolic genes. Previously, it has been reported that acH3K9 and acH3K27 could be activated by butyrate and enhance gene transcription [47, 48, 49]. In the previous studies, treatment of sodium butyrate accelerated adipogenesis and increased lipid accumulation by the hyperacetylation at the promoter regions of adipogenic genes such as PPAR $\gamma$ and C/EBP $[43,50]$. In the present study, ChIP results indicated that butyrate increased the amount of acH3K9 at the promoter of $\mathrm{C} / \mathrm{EBP} \beta$ and the amount of acH3K27 at the promoter of PPAR $\gamma$ and $\mathrm{C} /$ a

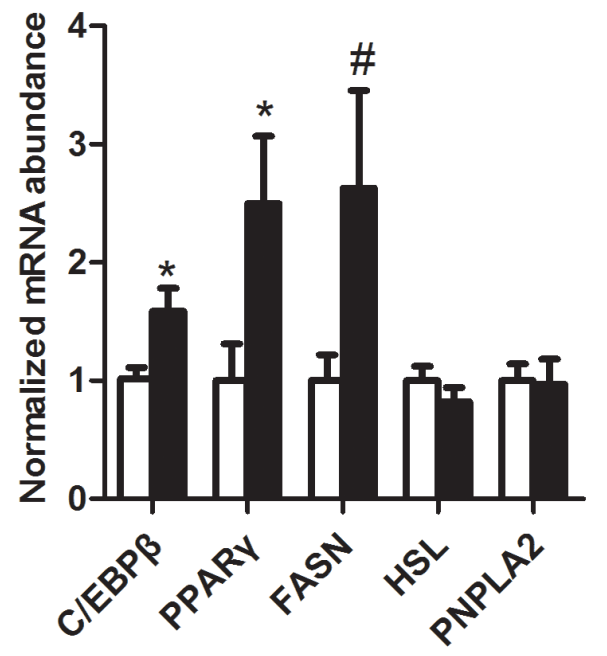

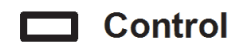

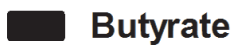

b
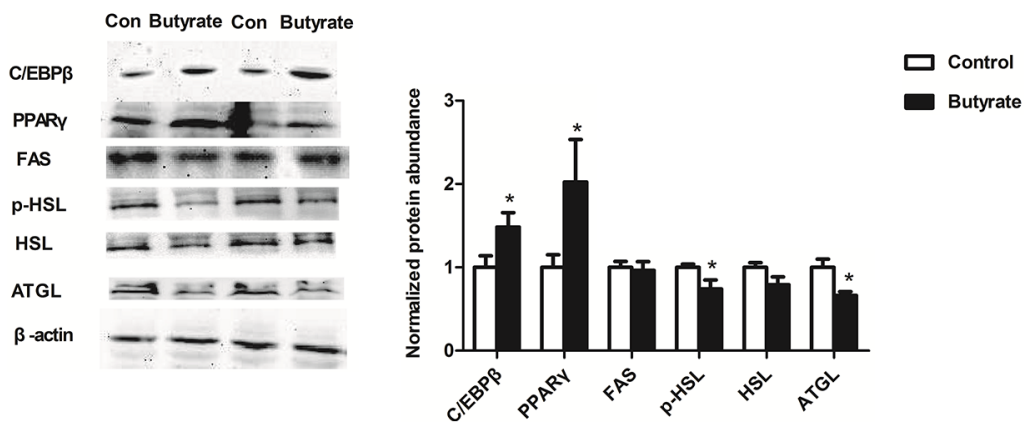

Figure 4: Effect of maternal butyrate supplementation throughout gestation and lactation on $\mathrm{mRNA}$ a. $(\mathrm{n}=8$ per group) and protein expression $b$. $(n=6$ per group) of genes related to lipid metabolism in skeletal muscle of adult offspring. $\beta$-actin was used for normalization. Values are mean \pm SEM. ${ }^{*} \mathrm{P}<0.05,{ }^{\sharp} \mathrm{P}<0.1$, compared with control. 
a

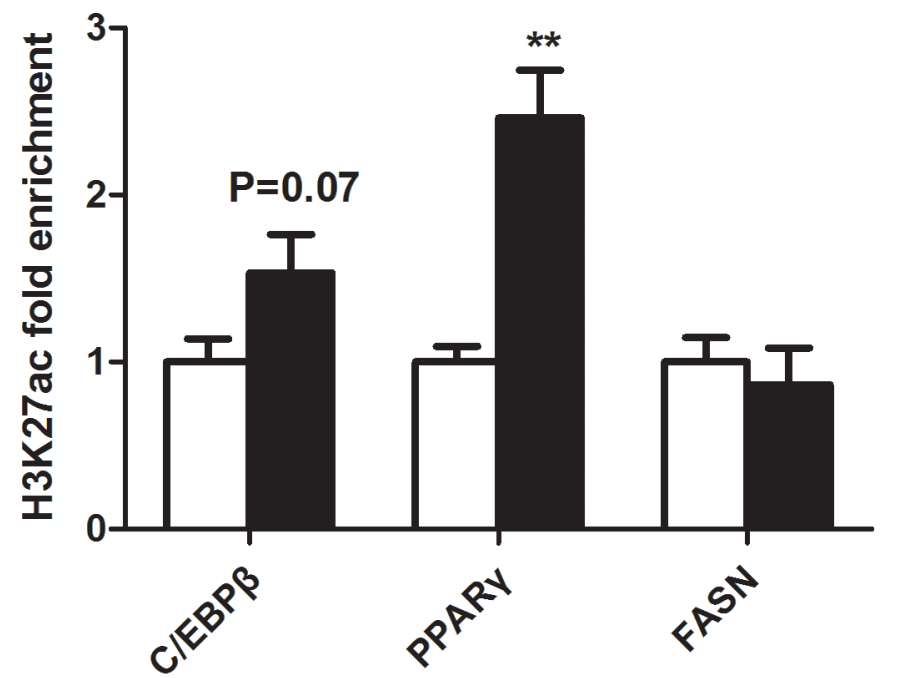

b

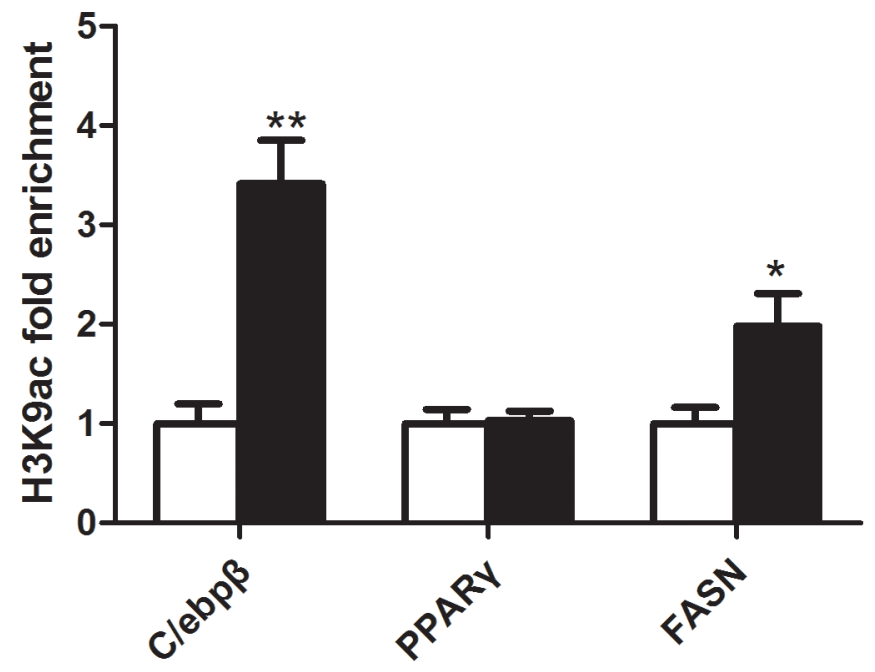

c

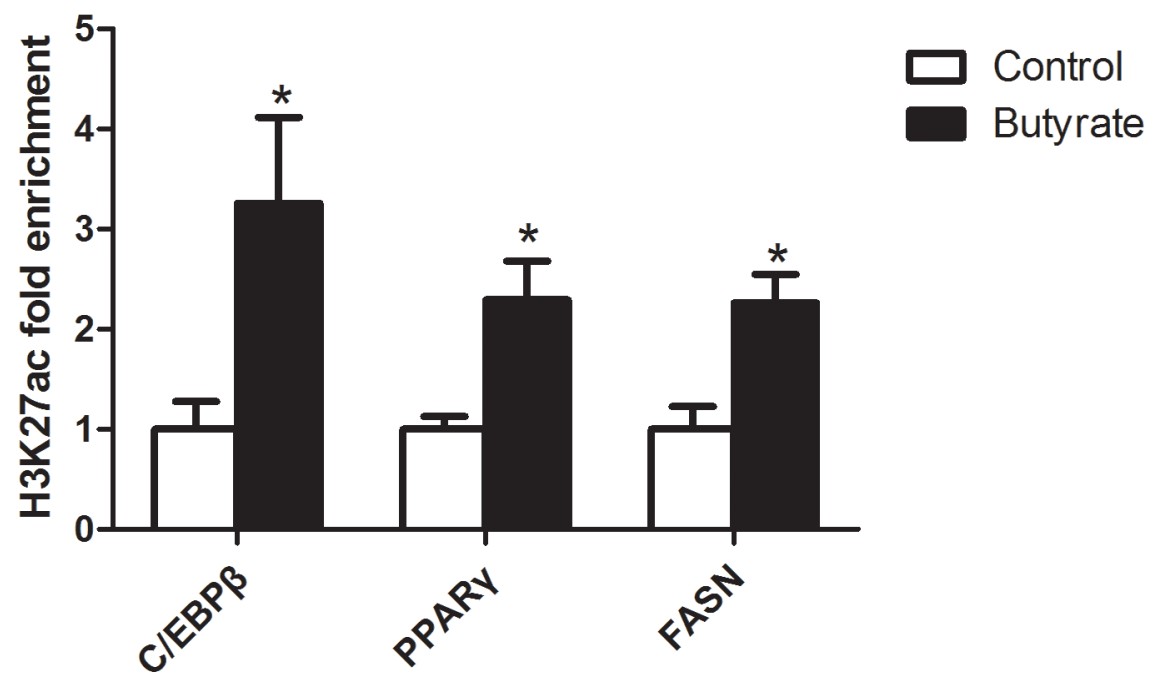

Figure 5: Effect of maternal butyrate supplementation throughout gestation and lactation on histone acetylation on the promoters of lipid- related genes in skeletal muscle. a. acH3K27 enrichment in skeletal muscle from offspring at weaning. b. acH3K9 enrichment in skeletal muscle from adult offspring. c. acH3K27 enrichment in skeletal muscle from adult offspring. Values are mean $\pm \operatorname{SEM}(\mathrm{n}=5$ or 6 per group). $* \mathrm{P}<0.05, * * \mathrm{P}<0.01$, compared with control. 
Table 3: Nutrition standard of experimental diets

\begin{tabular}{lcc}
\hline Ingredients (\%) & Control & Butyrate \\
\hline Corn & 47 & 46 \\
Wheat middling & 24 & 24 \\
Soya bean meal & 10 & 10 \\
Fish meal & 4 & 4 \\
Chicken meal & 5 & 5 \\
Premix & 4 & 4 \\
Salad oil & 1 & 1 \\
Limestone & 1 & 1 \\
Butyrate & 0 & 1 \\
Alfalfa & 4 & 4 \\
Total & 100 & 100 \\
Protein $(\%)$ & 22 & 22 \\
Fat $(\%)$ & 6.5 & 6.5 \\
Gross energy $(\mathrm{KJ} / \mathrm{g})$ & 17 & 17 \\
\hline
\end{tabular}

Table 4: Primer sequence for quantitative RT-PCR of mRNA

\begin{tabular}{lccc}
\hline Gene name & Primer sequence (5'-3') & Accession NO. & $\begin{array}{c}\text { Product } \\
\text { length(bp) }\end{array}$ \\
\hline HSL & F: CGCCTTACGGAGTCTATGC & 134 \\
R: TCTGATGGCTCTGAGTTGC & NM_012859 & 179 \\
FNPLA2 & R: GACCAACCCAACCCTTTG & NM_001108509 & 117 \\
PPAR $\gamma$ & F: TTGATTTCTCCAGCATTTC & & 92 \\
R: TGATCGCACTTTGGTATT & XM_006237009 & \\
F: GGGTTTCGGGACTTGATGC & & NM_001301715 & 218 \\
CD36 & R: GCCCGGCTGACAGTTACACG & & 195 \\
FASN & R: GTGCTCAACAGCCTTATC & NM_031561 & \\
& F:CTTAGTAGTGCGTGGTCGTAT & NM_017332 & \\
\hline
\end{tabular}

EBP $\beta$. These results support the possibility that butyrate stimulates lipogenesis in offspring skeletal muscle, in part through acetylation at the promoter regions of adipogenic genes. In this study, up-regulation of PPAR $\gamma$ gene was positive association with higher enrichment of acH3K27 on the promoter, in the skeletal muscle of butyrate group rats at weaning. Therefore, the increased amount of histone acetylation in the current research appears to play a role in the transcriptional regulation of lipogenic gene expression in skeletal muscle of offspring of butyrate supplemented rats. Follow-up studies in vitro are needed to identify the possible pathway in offspring after the maternal butyrate supplementation.

In conclusion, we are the first to demonstrate that a maternal butyrate diet during gestation and lactation leads to insulin resistance and accumulation of ectopic lipids in skeletal muscle of female offspring. This is associated with reductions in protein levels of key insulin signaling molecules and increase in protein expressions of molecules involved in lipid accumulation in skeletal 
Table 5: Primer sequence for the promoters

\begin{tabular}{llc}
\hline Gene name & Primer sequence (5'-3') & Product length(bp) \\
\hline PPAR $\gamma$ & F: TAGGGTGGAAGGACATG & 160 \\
C/EBP $\beta$ & R: GTTGGGAGACAGGGAAT & 115 \\
& F: GGTGACCGTTGCGTCCTT & 111 \\
FASN & R: GGTAGTGGTGTCCGGGTATCA & \\
\hline
\end{tabular}

muscle. These findings contribute to a better understanding of mechanisms that mediate the effects of early nutrition on the risk of developing type 2 diabetes.

\section{MATERIALS AND METHODS}

\section{Experimental design and sampling}

Twenty-eight virgin female Sprague Dawley rats were purchased from Laboratory Animal Centre, university of Jiangsu and housed in individual cages under temperature- and humidity controlled conditions with a 12-h light/dark cycle. After $7 \mathrm{~d}$ of acclimatization, rats were mated, and conception was confirmed by the presence of spermatozoids in the vaginal wash. Pregnant rats were randomly assigned to either control or butyrate diet ( $1 \%$ butyrate sodium), with water ad libitum. The control diet was prepared following the recommendations of the American Institute of Nutrition for rodent growth (AIN-93G) (Table 3).

After delivering, litters were adjusted to eight animals for each dam, maintaining the sex ratio as close as possible to $1: 1$. During lactation, dams continued to consume their assigned experimental diet. Eight female offspring of either group were randomly selected and killed at 21 days of age (weaning). Further 8 animals per group were fed with the same control diet and killed at 60 days of age. Animals were anesthetized with pentobarbital sodium and sacrificed by bleeding. A blood sample was collected, the gastrocnemius muscles were excised, weighed, immediately snap frozen in liquid nitrogen and stored at $-80^{\circ} \mathrm{C}$ until analysis.

All animal procedures were approved by the Institutional Animal Care and Use Committee (IACUC) of Nanjing Agricultural University. The protocol of this study was reviewed and approved with the project number 2012CB124703. The slaughter and sampling procedures strictly followed the "Guidelines on Ethical Treatment of Experimental Animals" (2006) No. 398 set out by the Ministry of Science and Technology, China and the Regulation regarding the Management and Treatment of Experimental Animals" (2008) No. 45 set out by the Jiangsu Provincial People's Government.

\section{Biochemical analysis}

Serum concentration of glucose, total cholesterol, total triglyceride, HDL-cholesterol (HDL - C), LDLcholesterol (LDL - C) and non-esterified fatty acid (NEFA) were measured by biochemical automatic analyzer (Hitachi 7020, HITACHI, Tokyo, Japan) using commercial assay kits (KH674, KQ436, AM545, KP712 and KF253, respectively; Wako Pure Chemical Industries, Ltd. Wako; Japan). Total triglyceride concentration in skeletal muscle (Intramuscular Triglyceride) was measured using a tissue total triglyceride assay kit (E1013; Applygen Technologies, Inc.) following the manufacturer's instructions.

Butyrate levels in the serum of the dams were published in our previous study $(32.9 \pm 2.9 \mu \mathrm{M}$ vs $63.7 \pm 12.3 \mu \mathrm{M} ; \mathrm{P}<0.05)$. It could be detected by gas chromatographic analysis. The extraction and gas chromatographic analysis procedure was carried out as described by Zhao et al. [29] with some modifications.

\section{Metabolic assays}

Intraperitoneal glucose tolerance test (GTT) $(n=10$ in controls; $\mathrm{n}=12$ in butyrate group) and insulin tolerance test (ITT) ( $\mathrm{n}=5$ per group) were performed in overnight fasted rats. Blood samples were obtained from the tail tip at $0,15,30,45,60,90,120 \mathrm{~min}$ for GTT. For ITT, blood samples were collected at $0,15,30,45,60 \mathrm{~min}$. Glucose levels were measured using a glucometer (AccuCheck II; Roche, Castle Hill, NSW, Australia). The doses used during these tests were $1.5 \mathrm{~g} / \mathrm{kg}$ body weight and $0.5 \mathrm{U} / \mathrm{kg}$ body weight for GTT and ITT, respectively.

\section{RNA isolation and real-time PCR for mRNA quantification}

Total RNA was isolated from gastrocnemius muscles ( $\mathrm{n}=8$ per group) with TRIzol Reagent (Invitrogen Life Technologies, USA) and reverse transcribed with the PrimeScript 1st Strand cDNA Synthesis Kit (RR048A, Takara, Tokyo, Japan) according to the manufacturer's instructions. Diluted cDNA $(2 \mu 1,1: 25)$ was used in each real-time PCR assay with Mx3000P (Stratagene, Agilent Technologies, USA and CA). All primers (Table 4 and 5) 
were synthesized by Generay Biotech (Shanghai, China). For normalization, $\beta$-actin was chosen as a reference gene and was not affected by the experimental factors.

\section{Protein extraction and Western blotting analysis}

Total cellular protein was extracted from $100 \mathrm{mg}$ of frozen gastrocnemius muscle ( $n=6$ per group) samples as described previously [29]. Protein concentrations were measured with a Pierce BCA Protein Assay kit (no. 23225, Thermo Scientific). Western blot analysis of target proteins was carried out according to the protocols provided by the manufacturer. The sources of primary antibodies used in Western blot were as follows: anti- PPAR $\gamma$ (AP0686, Bioworld Technology, Nanjing, China), anti-C/EBP $\beta$ (sc150X, Santa Cruz Biotechnology, Santa Cruz, CA, USA), anti-phosphor-ser ${ }^{855}$ HSL (P-HSL, BS4234, Bioworld Technology); anti-HSL (BS2742, Bioworld Technology); anti-ATGL (BS7989, Bioworld Technology); anti- GLUT4 (sc53566, Santa Cruz Biotechnology); antiphosphor-ser ${ }^{1101}$ IRS (BS4239P, Bioworld Technology); anti-IRS (BS3589, Bioworld Technology); anti-PI3K p85 $\alpha$ (BS3678, Bioworld Technology); anti-AKT (AP0059, Bioworld Technology); anti-PKC $\theta$ (BS3666, Bioworld Technology); anti-Histone 3acetyl K9 (ab10812, abcam, Cambridge, UK); anti-Histone 3acetyl K27 (ab4729, abcam); $\beta$-actin (KC5AO8, Kangcheng, Nanjing, China).

\section{Chromatin immunoprecipitation assay}

Approximately $200 \mathrm{mg}$ of frozen gastrocnemius muscle samples ( $n=5$ or 6 per group) were ground in liquid $\mathrm{N}_{2}$ and washed with PBS containing protease inhibitor cocktail (no. 11697498001; Roche; Castle Hill, NSW, Australia). Cross-linking of protein and DNA was performed by adding formaldehyde to a final concentration of $1 \%$, and then the reaction was stopped with glycine $(2.5 \mathrm{~mol} / \mathrm{l})$ at room temperature. The reaction mixture was centrifuged and the pellets were washed with PBS and lysed in a SDS lysis buffer. Chromatin was sonicated to an average length ranging from 200 to 500 bp and precleared with protein $\mathrm{G}$ agarose beads treated with salmon sperm DNA (40 $\mu 1,50 \%$ slurry, sc-2003; Santa Cruz Biotechnology). The mixture of chromatin preparations and $2 \mu \mathrm{g}$ of specific primary antibody were incubated overnight at $4^{\circ} \mathrm{C}$. A negative control was included with normal rat IgG. Protein $\mathrm{G}$ agarose beads (40 $\mu 1,50 \%$ slurry, sc-2003; Santa Cruz Biotechnology) were added to capture the immunoprecipitated chromatin complexes. Finally, reverse cross-linking was performed to release DNA fragments from the immunoprecipitated complex at $65^{\circ} \mathrm{C}$ for $1 \mathrm{~h}$, and quantitative real-time PCR was used to quantify the fragments of target gene promoters with specific primers (Table 5) using purified immunoprecipitated DNA as the template.

\section{Statistical analysis}

All data are presented as the mean \pm SEM. The statistical analyses were performed using the Statistical Program for Social Sciences (SPSS) software 18.0 for Windows (SPSS Inc., Chicago, IL, USA). The $2^{-\Delta \Delta \mathrm{Ct}}$ method was applied to analyze real-time PCR data. The differences were tested with analysis of variance (ANOVA), and a t-test was used for the independent samples. Differences were considered significant at $P<0.05$.

\section{Abbreviations}

AKT, Protein kinase B; ATGL, Adipose triglyceride lipase; C/EBP, CCAAT/enhancer-binding protein; GLUT4, Glucose transporter 4; HDL-C, HDLcholesterol; HSL, Hormone sensitive lipase; IRS, Insulin receptor substrate; LDL-C, LDL-cholesterol; NEFA, Non esterifiable fatty acid; PI3K, Phosphatidylinositol 3-kinase; PKC $\theta$, Protein kinase C $\theta$; PPAR $\gamma$, Peroxisome proliferator-activated receptor $\gamma$

\section{ACKNOWLEDGMENTS}

We would like to acknowledge the Priority Academic Program Development of Jiangsu Higher Education Institutions. This study was supported by the National Natural Science Foundation of China (31572482) and the National Key Research and Development Program of China (2016YFD0500502).

\section{CONFLICTS OF INTEREST}

The authors declare that there is no conflict of interest associated with this manuscript.

\section{Author contributions}

The authors' contributions were as follows: all authors contributed to the study conception and design; YH performed the experiments and analyzed the data and wrote the draft manuscript; SG and JC performed animals keeping and the GTT and ITT; RZ and XY provided scientific direction and interpreted the results; EA revised and XY finalized the manuscript. All authors have given final approval of the manuscript. XY is responsible for the integrity of the work as a whole.

\section{REFERENCES}

1. Mennitti LV, Oliveira JL, Morais CA, Estadella D, Oyama LM, Oller do Nascimento CM, Pisani LP. Type of fatty acids in maternal diets during pregnancy and/or lactation 
and metabolic consequences of the offspring. The Journal of nutritional biochemistry. 2015; 26:99-111.

2. Godfrey KM, Barker DJ. Fetal programming and adult health. Public health nutrition. 2001; 4:611-624.

3. Alfaradhi MZ, Ozanne SE. Developmental programming in response to maternal overnutrition. Frontiers in genetics. 2011; doi: 10.3389/fgene.2011.00027.

4. Ainge H, Thompson C, Ozanne SE, Rooney KB. A systematic review on animal models of maternal high fat feeding and offspring glycaemic control. Int J Obes (Lond). 2011; 35:325-335.

5. DeFronzo RA. Insulin resistance, lipotoxicity, type 2 diabetes and atherosclerosis: the missing links. The Claude Bernard Lecture 2009. Diabetologia. 2010; 53:1270-1287.

6. Symonds ME, Sebert SP, Hyatt MA, Budge H. Nutritional programming of the metabolic syndrome. Nat Rev Endocrinol. 2009; 5:604-610.

7. Shulman GI. Cellular mechanisms of insulin resistance. J Clin Invest. 2000; 106:171-176.

8. Innis SM. Metabolic programming of long-term outcomes due to fatty acid nutrition in early life. Matern Child Nutr. 2011; 7:112-123.

9. Boffa LC, Vidali G, Mann RS, Allfrey VG. Suppression of histone deacetylation in vivo and in vitro by sodium butyrate. The Journal of biological chemistry. 1978; 253:3364-3366

10. Yen JT, Nienaber JA, Hill DA, Pond WG. Potential Contribution of Absorbed Volatile Fatty-Acids to WholeAnimal Energy Requirement in Conscious Swine. Journal of animal science. 1991; 69:2001-2012.

11. Sicilianojones J, Murphy MR. Production of Volatile FattyAcids in the Rumen and Cecum-Colon of Steers as Affected by Forage - Concentrate and Forage Physical Form. Journal of dairy science. 1989; 72:485-492.

12. Bramswig NC, Kaestner KH. Epigenetics and diabetes treatment: an unrealized promise? Trends in endocrinology and metabolism: TEM. 2012; 23:286-291.

13. Lee HB, Noh H, Seo JY, Yu MR, Ha H. Histone deacetylase inhibitors: a novel class of therapeutic agents in diabetic nephropathy. Kidney international Supplement. 2007; S61-66.

14. Gao Z, Yin J, Zhang J, Ward RE, Martin RJ, Lefevre M, Cefalu WT, Ye J. Butyrate improves insulin sensitivity and increases energy expenditure in mice. Diabetes. 2009; 58:1509-1517.

15. Hu GX, Chen GR, Xu H, Ge RS, Lin J. Activation of the AMP activated protein kinase by short-chain fatty acids is the main mechanism underlying the beneficial effect of a high fiber diet on the metabolic syndrome. Medical hypotheses. 2010; 74:123-126.

16. Lan X, Cretney EC, Kropp J, Khateeb K, Berg MA, Penagaricano F, Magness R, Radunz AE, Khatib H. Maternal Diet during Pregnancy Induces Gene Expression and DNA Methylation Changes in Fetal Tissues in Sheep. Frontiers in genetics. 2013; 4:49.

17. Radunz AE, Fluharty FL, Relling AE, Felix TL, Shoup LM, Zerby HN, Loerch SC. Prepartum dietary energy source fed to beef cows: II. Effects on progeny postnatal growth, glucose tolerance, and carcass composition. Journal of animal science. 2012; 90:4962-4974.

18. Yan H, Ajuwon KM. Mechanism of Butyrate Stimulation of Triglyceride Storage and Adipokine Expression during Adipogenic Differentiation of Porcine Stromovascular Cells. PloS one. 2015; 10: e0145940.

19. Li G, Yao W, Jiang H. Short-chain fatty acids enhance adipocyte differentiation in the stromal vascular fraction of porcine adipose tissue. The Journal of nutrition 2014; 144:1887-1895.

20. Itani SI, Ruderman NB, Schmieder F, Boden G. Lipidinduced insulin resistance in human muscle is associated with changes in diacylglycerol, protein kinase $\mathrm{C}$, and I kappa B-alpha. Diabetes. 2002; 51:2005-2011.

21. Li YX, Han TT, Liu Y, Zheng S, Zhang Y, Liu W, Hu YM. Insulin resistance caused by lipotoxicity is related to oxidative stress and endoplasmic reticulum stress in LPL gene knockout heterozygous mice. Atherosclerosis. 2015; 239:276-282.

22. De Stefanis D, Mastrocola R, Nigro D, Costelli P, Aragno M. Effects of chronic sugar consumption on lipid accumulation and autophagy in the skeletal muscle. European journal of nutrition. 2015; doi:10.1007/s00394-015-1086-8.

23. Badin PM, Vila IK, Louche K, Mairal A, Marques MA, Bourlier V, Tavernier G, Langin D, Moro C. High-Fat DietMediated Lipotoxicity and Insulin Resistance Is Related to Impaired Lipase Expression in Mouse Skeletal Muscle. Endocrinology. 2013; 154:1444-1453.

24. Goodpaster BH, Kelley DE. Skeletal muscle triglyceride: marker or mediator of obesity-induced insulin resistance in type 2 diabetes mellitus? Current diabetes reports. 2002; 2:216-222.

25. Muoio DM. Intramuscular triacylglycerol and insulin resistance: guilty as charged or wrongly accused? Biochimica et biophysica acta. 2010; 1801:281-288.

26. Pan DA, Lillioja S, Kriketos AD, Milner MR, Baur LA, Bogardus C, Jenkins AB, Storlien LH. Skeletal muscle triglyceride levels are inversely related to insulin action. Diabetes. 1997; 46:983-988.

27. Phillips DI, Caddy S, Ilic V, Fielding BA, Frayn KN, Borthwick AC, Taylor R. Intramuscular triglyceride and muscle insulin sensitivity: evidence for a relationship in nondiabetic subjects. Metabolism: clinical and experimental. 1996; 45:947-950.

28. Storlien LH, Jenkins AB, Chisholm DJ, Pascoe WS, Khouri $\mathrm{S}$, Kraegen EW: Influence of dietary fat composition on development of insulin resistance in rats. Relationship to muscle triglyceride and omega- 3 fatty acids in muscle phospholipid. Diabetes. 1991;40:280-289. 
29. Zhao, G, Liu, JF, Nyman, M, Jonsson, JA. Determination of short-chain fatty acids in serum by hollow fiber supported liquid membrane extraction coupled with gas chromatography. Journal of chromatography. B, Analytical technologies in the biomedical and life sciences. 2007; 846: 202-208.

30. Latouche C, Heywood SE, Henry SL, Ziemann M, Lazarus R, El-Osta A, Armitage JA, Kingwell BA. Maternal Overnutrition Programs Changes in the Expression of Skeletal Muscle Genes That Are Associated with Insulin Resistance and Defects of Oxidative Phosphorylation in Adult Male Rat Offspring. Journal of Nutrition. 2014; 144:237-244.

31. Bayol SA, Simbi BH, Stickland NC. A maternal cafeteria diet during gestation and lactation promotes adiposity and impairs skeletal muscle development and metabolism in rat offspring at weaning. J Physiol-London. 2005; 567:951-961.

32. DeFronzo RA, Tripathy D. Skeletal Muscle Insulin Resistance Is the Primary Defect in Type 2 Diabetes. Diabetes Care. 2009; 32:157-163.

33. Martin-Gronert MS, Fernandez-Twinn DS, Bushell M, Siddle K, Ozanne SE. Cell-autonomous programming of rat adipose tissue insulin signalling proteins by maternal nutrition. Diabetologia. 2016; 59:1266-75.

34. Zhu SM, Sun F, Li WJ, Cao YJ, Wang C, Wang YB, Liang D, Zhang RQ, Zhang SW, Wang HC, Cao F. Apelin stimulates glucose uptake through the PI3K/Akt pathway and improves insulin resistance in 3T3-L1 adipocytes. Mol Cell Biochem. 2011; 353:305-313.

35. Li Y, Soos TJ, Li XH, Wu J, DeGennaro M, Sun XJ, Littman DR, Birnbaum MJ, Polakiewicz RD. Protein kinase $C$ theta inhibits insulin signaling by phosphorylating IRS1 at Ser(1101). Journal of Biological Chemistry. 2004; 279:45304-45307.

36. Nicholas LM, Morrison JL, Rattanatray L, Ozanne SE, Kleemann DO, Walker SK, MacLaughlin SM, Zhang S, Martin-Gronert MS, McMillen IC. Differential Effects of Exposure to Maternal Obesity or Maternal Weight Loss during the Periconceptional Period in the Sheep on Insulin Signalling Molecules in Skeletal Muscle of the Offspring at 4 Months of Age. PloS one. 2013; 8: e84594.

37. Zhou J, Gao S, Chen J, Zhao R, Yang X. Maternal sodium butyrate supplement elevates the lipolysis in adipose tissue and leads to lipid accumulation in offspring liver of weaning-age rats. Lipids Health Dis. 2016; 15, 119.

38. Kim TH, Kim MY, Jo SH, Park JM, Ahn YH. Modulation of the Transcriptional Activity of Peroxisome
Proliferator-Activated Receptor Gamma by Protein-Protein Interactions and Post-Translational Modifications. Yonsei Med J. 2013; 54:545-559.

39. Ilavenil S, Arasu MV, Lee JC, Kim DH, Vijayakumar M, Lee KD, Choi KC. Positive regulations of adipogenesis by Italian ryegrass [Lolium multiflorum] in 3T3-L1 cells. Bmc Biotechnol. 2014; 14.

40. White UA, Stephens JM. Transcriptional factors that promote formation of white adipose tissue. Mol Cell Endocrinol. 2010; 318:10-14.

41. Farmer SR. Transcriptional control of adipocyte formation. Cell metabolism. 2006; 4:263-273.

42. Siersbaek R, Nielsen R, Mandrup S. Transcriptional networks and chromatin remodeling controlling adipogenesis. Trends Endocrin Met. 2012; 23:56-64.

43. Yoo EJ, Chung JJ, Choe SS, Kim KH, Kim JB. Downregulation of histone deacetylases stimulates adipocyte differentiation. The Journal of biological chemistry. 2006; 281:6608-6615.

44. Jordy AB, Kiens B. Regulation of exercise-induced lipid metabolism in skeletal muscle. Experimental physiology. 2014; 99:1586-1592.

45. Badin PM, Louche K, Mairal A, Liebisch G, Schmitz G, Rustan AC, Smith SR, Langin D, Moro C. Altered skeletal muscle lipase expression and activity contribute to insulin resistance in humans. Diabetes. 2011; 60:1734-1742.

46. Mathew OP, Ranganna K, Yatsu FM. Butyrate, an HDAC inhibitor, stimulates interplay between different posttranslational modifications of histone $\mathrm{H} 3$ and differently alters G1-specific cell cycle proteins in vascular smooth muscle cells. Biomed Pharmacother. 2010; 64:733-740.

47. Kumar P, Periyasamy R, Das S, Neerukonda S, Mani I, Pandey KN. All-Trans Retinoic Acid and Sodium Butyrate Enhance Natriuretic Peptide Receptor A Gene Transcription: Role of Histone Modification. Mol Pharmacol. 2014; 85:946-957.

48. Shin JH, Li RW, Gao Y, Baldwin Rt, Li CJ. Genomewide ChIP-seq mapping and analysis reveal butyrateinduced acetylation of $\mathrm{H} 3 \mathrm{~K} 9$ and $\mathrm{H} 3 \mathrm{~K} 27$ correlated with transcription activity in bovine cells. Functional \& integrative genomics. 2012; 12:119-130.

49. Zhou VW, Goren A, Bernstein BE. Charting histone modifications and the functional organization of mammalian genomes. Nature reviews Genetics. 2011; 12:7-18.

50. Kim SN, Choi HY, Kim YK. Regulation of adipocyte differentiation by histone deacetylase inhibitors. Arch Pharm Res. 2009; 32:535-541. 